\title{
Effect of Moisture Content on High Strain Rate Compressibility and Particle Breakage in Loose Sand
}

\author{
A. D. Barr ${ }^{1}$ (I) . S. D. Clarke ${ }^{1}$ A. Tyas ${ }^{1,2} \cdot$ J. A. Warren ${ }^{1,2}$ \\ Received: 26 February 2018 / Accepted: 21 May 2018 / Published online: 14 June 2018 \\ (C) The Author(s) 2018
}

\begin{abstract}
Soil-filled gabion structures are widely used to protect against the effects of blast and fragmentation. It is known that moisture content significantly affects the capability of such structures, but the behaviour of partially-saturated soils is not well characterised at the strain rates and stresses experienced in these events. In particular, little data is available for loose soils, whose compaction behaviour can have a substantial impact on structural stability and ballistic performance. This paper describes the use of split Hopkinson pressure bar experiments to characterise the pre- and post-saturation compressibility of a loose quartz sand at moisture contents of up to $15.0 \%$. In contrast to dense soils, increases in moisture content between $0.0 \%$ and $7.5 \%$ led to a decrease in the stiffness of the sand. Above $7.5 \%$ moisture content, specimens reached full saturation during the experiment: the additional water had no further effect on the pre-saturation stiffness, but post-saturation behaviour was dominated by the stiffness of the pore water. Full saturation occurred at lower dry densities as moisture content increased, leading to a decrease in particle breakage.
\end{abstract}

Keywords Split Hopkinson pressure bar $\cdot$ Soil $\cdot$ Sand $\cdot$ Moisture content $\cdot$ Compressibility $\cdot$ Particle breakage $\cdot$ Strain rate

The split Hopkinson pressure bar (SHPB) has been used by a number of authors to investigate the effect of moisture content on the high-strain-rate behaviour of soils. Felice et al. [1], Veyera [2] and Luo et al. [3] performed experiments on dense sandy soils at strain rates between $600 \mathrm{~s}^{-1}$ and $2000 \mathrm{~s}^{-1}$ over a wide range of moisture contents. As the soils were all dense, little variation in pre-saturation behaviour was observed, although the soils stiffened rapidly on reaching full saturation. Luo et al. [3] additionally observed that particle breakage decreased as moisture content increased in specimens reaching saturation. In experiments on a loose sand by Martin et al. [4] all partially-saturated specimens behaved less stiffly than dry specimens, although there seemed to be no significant difference between the partially-saturated specimens, with considerable overlap between test series. These tests were

A. D. Barr

a.barr@sheffield.ac.uk

1 Department of Civil and Structural Engineering, The University of Sheffield, Sheffield, S1 3JD, UK

2 Blastech Ltd., The BioIncubator, 40 Leavygreave Road, Sheffield, S3 7RD, UK conducted at relatively low axial stresses (15 MPa), so full saturation did not occur. Barr et al. [5] tested a loose sand at moisture contents between $0.0 \%$ and $5.0 \%$. None of the specimens reached full saturation during loading to $400 \mathrm{MPa}$, and no significant variation in stiffness was observed, although the authors also noted that the behaviour of the specimens was dominated by inertial effects. Modelling of soil-filled gabions in blast or impact events requires an understanding of the dynamic behaviour of relatively loose soils at high stresses, where the early compressibility of the soil can significantly affect both the stability and ballistic resistance of the structure $[6,7]$. However, the majority of the existing work is concerned with either dense soils [1-3] or loose soils at low stresses [4]. This paper considers the behaviour of loose quartz sand loaded to $200 \mathrm{MPa}$ at moisture contents between $0.0 \%$ and $15.0 \%$, providing information on the effects of moisture content on both pre- and post-saturation stiffness.

The soil tested was a uniform fine and medium quartz sand (EN ISO 14688-1:2002), with a mean particle size of $250 \mu \mathrm{m}$ and a coefficient of uniformity of 2.2 [5]. The initial particle size distribution (PSD) shown in Fig. 4 was assessed using a Malvern Mastersizer 3000 particle size analyser with a wet dispersion unit: all PSDs in this 
Table 1 Initial saturation ratios and dry density required to reach full saturation for moisture contents between $0.0 \%$ and $15.0 \%$

\begin{tabular}{lllrrrrc}
\hline Moisture content $(\%)$ & 0.0 & 2.5 & 5.0 & 7.5 & 10.0 & 12.5 & 15.0 \\
Saturation ratio at $\rho_{d}=1.5 \mathrm{Mg} / \mathrm{m}^{3}(\%)$ & 0.0 & 8.6 & 17.3 & 25.9 & 34.6 & 43.2 & 51.8 \\
Dry density at full saturation $\left(\mathrm{Mg} / \mathrm{m}^{3}\right)$ & - & 2.49 & 2.34 & 2.21 & 2.09 & 1.99 & 1.90 \\
\hline
\end{tabular}

paper are represented by a curve which encloses the PSD histogram, which is formed of 50 logarithmically-spaced bins. The sand has minimum and maximum dry densities of $\rho_{\mathrm{d}, \min }=1.35 \mathrm{Mg} / \mathrm{m}^{3}$ and $\rho_{\mathrm{d}, \max }=1.87 \mathrm{Mg} / \mathrm{m}^{3}$, and was prepared at a loose dry density of $\rho_{d}=1.5 \mathrm{Mg} / \mathrm{m}^{3}$ (density index, $\left.I_{D}=0.35\right)$. Specimens were prepared at moisture contents of $0.0 \%$ to $15.0 \%$ in $2.5 \%$ increments as listed in Table 1, which also shows the initial saturation ratios. Three specimens were tested at each moisture content using a constant dry mass of $3.50 \mathrm{~g}$, and with a nominal diameter of $25 \mathrm{~mm}$ and length of $4.8 \mathrm{~mm}$.

The SHPB used by Barr et al. [5] was modified to provide a single specimen loading by using a transmitter bar less than half the length of the incident bar, so that the transmitter bar acted as a momentum trap [8]. The dimensions of the 440C stainless steel bars are shown in Fig. 1a. Specimens were loaded in uniaxial strain using a steel confining ring [5]: this limited radial strains to $0.05 \%$ during experiments with axial strains exceeding $30 \%$. A lidded polypropylene box was fitted around the confining ring assembly to ensure that the sand specimens were retained for further study (Fig. 1b). Strain data was recorded using semiconductor strain gauges and a TiePie Handyscope oscilloscope as described by Barr et al. [5]. Axial stresses were calculated using a frequency-domain dispersion correction algorithm using the stresses from both pressure bars, and so represent the mean axial stress in the specimen $[9,10]$.

Specimens achieved peak axial stresses of approximately $200 \mathrm{MPa}$ and peak strain rates of $3000 \mathrm{~s}^{-1}$, as shown in Fig. 2. The application of pulse shaping to experiments on soils has not yet been successfully demonstrated at these stresses and strain rates, and so has not been applied here. As a result, the stress on the front face of the specimen is considerably higher than on the back face for the first $70 \mu \mathrm{s}$ in Fig. 2a, indicating that inertial forces dominate in this region and the material behaviour may not be accurately represented. The first $70 \mu$ s corresponds to compression up to approximately $1.8 \mathrm{Mg} / \mathrm{m}^{3}$ in Fig. 3, and so the comments below relate to the portion of the loading above $1.8 \mathrm{Mg} / \mathrm{m}^{3}$, where dynamic equilibrium has been achieved and meaningful comparisons can be made.

The mean axial stress-dry density behaviour in Fig. 3a shows that for moisture contents between $0.0 \%$ and $7.5 \%$ the stiffness of the soil specimen decreased as the moisture content increased. These specimens remained partiallysaturated throughout the experiment (Table 1), and so the reduction in stiffness appears to indicate a reduction in interparticle friction: this behaviour is similar to that observed in SHPB experiments by Martin et al. [4], but to axial stresses of $200 \mathrm{MPa}$. Figure $4 \mathrm{a}$ shows the mean pre- and post-test PSDs of the $0.0 \%$ to $7.5 \%$ moisture content specimens, where the particle breakage increased slightly with moisture content. This appears to be related to the larger strains obtained at higher moisture contents, where the reduction in inter-particle friction would increase the energy available for further breakage and compaction.

For moisture contents of $10.0 \%$ to $15.0 \%$ the sand reached full saturation during loading (Fig. 3b). Prior to full saturation, the behaviour at $10.0 \%$ to $15.0 \%$ moisture content is very similar to the $7.5 \%$ specimens, showing no additional reduction in stiffness with increased moisture content. On reaching saturation the increase in stiffness corresponded closely to the EOS of water in each case (dashed lines in Fig. 3b), indicating that subsequent volume changes were dominated by the compression of the pore water. Figure $4 \mathrm{~b}$ shows the mean pre- and post-test PSDs of the $10.0 \%$ to $15.0 \%$ moisture content specimens, where particle breakage decreased significantly as the moisture content increased. This result agrees with the experiments on dense soils carried out by Luo et al. [3]. On

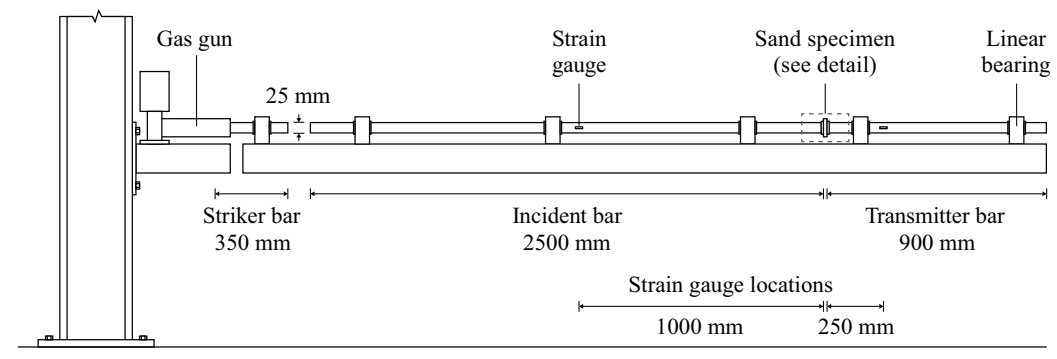

(a)

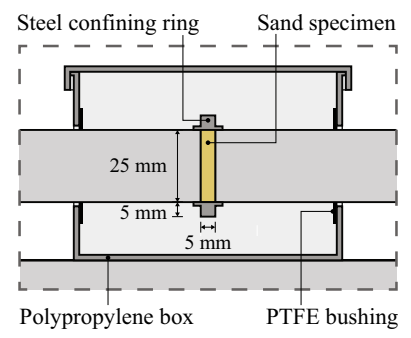

(b)

Fig. 1 a Dimensions of modified split Hopkinson pressure bar for single specimen loading, and $\mathbf{b}$ section detail of specimen confinement 

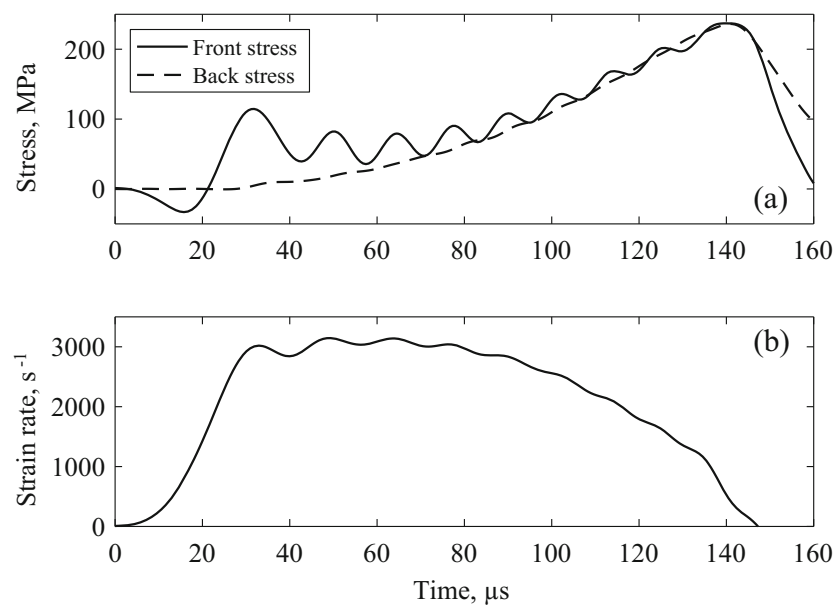

Fig. 2 Typical a axial stress and $\mathbf{b}$ strain rate histories in the current experiments
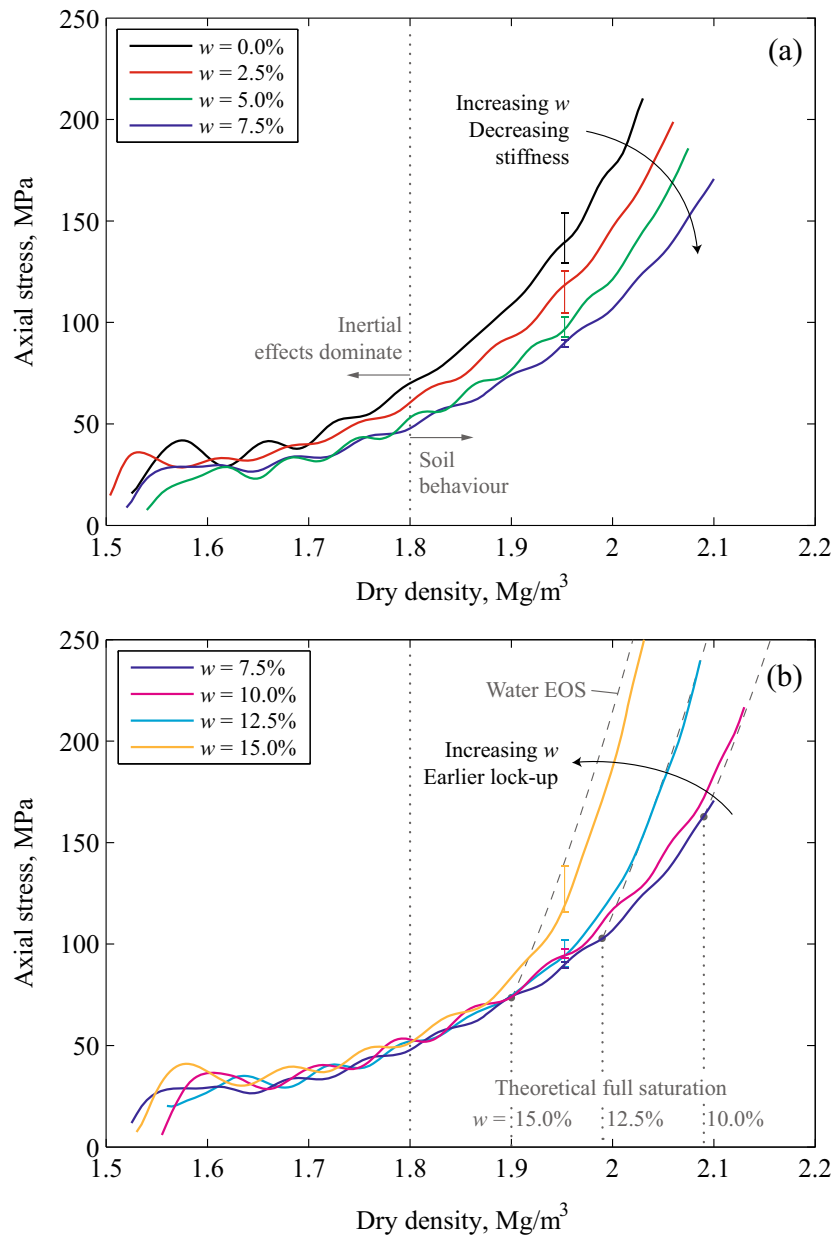

Fig. 3 Mean axial stress-dry density results for moisture contents of a $0.0 \%$ to $7.5 \%$, remaining partially-saturated throughout, and b $10.0 \%$ to $15.0 \%$, reaching full saturation during loading $(7.5 \%$ results repeated for reference). Error bars indicate full range of results. EOS of water from Shin et al. [11] reaching full saturation the sand particles moved from being loaded mainly via particle-to-particle contact to include a significant hydrostatic component from the surrounding pore water. The resulting decrease in deviatoric stress would make particle breakage less likely, and so the majority of breakage should occur before the specimen reaches full saturation. This is confirmed through comparison of the $7.5 \%$ and $10.0 \%$ specimens, as the $7.5 \%$ specimens achieve a dry density of approximately $2.10 \mathrm{Mg} / \mathrm{m}^{3}$, which is the point at which the $10.0 \%$ specimens reach full saturation. Through compression of the pore water the $10.0 \%$ specimens continue to a dry density of nearly $2.15 \mathrm{Mg} / \mathrm{m}^{3}$, but have a post-test PSD which is very similar to the $7.5 \%$ specimens. Full saturation occurred at lower stresses as moisture content increased, resulting in a reduction in particle breakage.
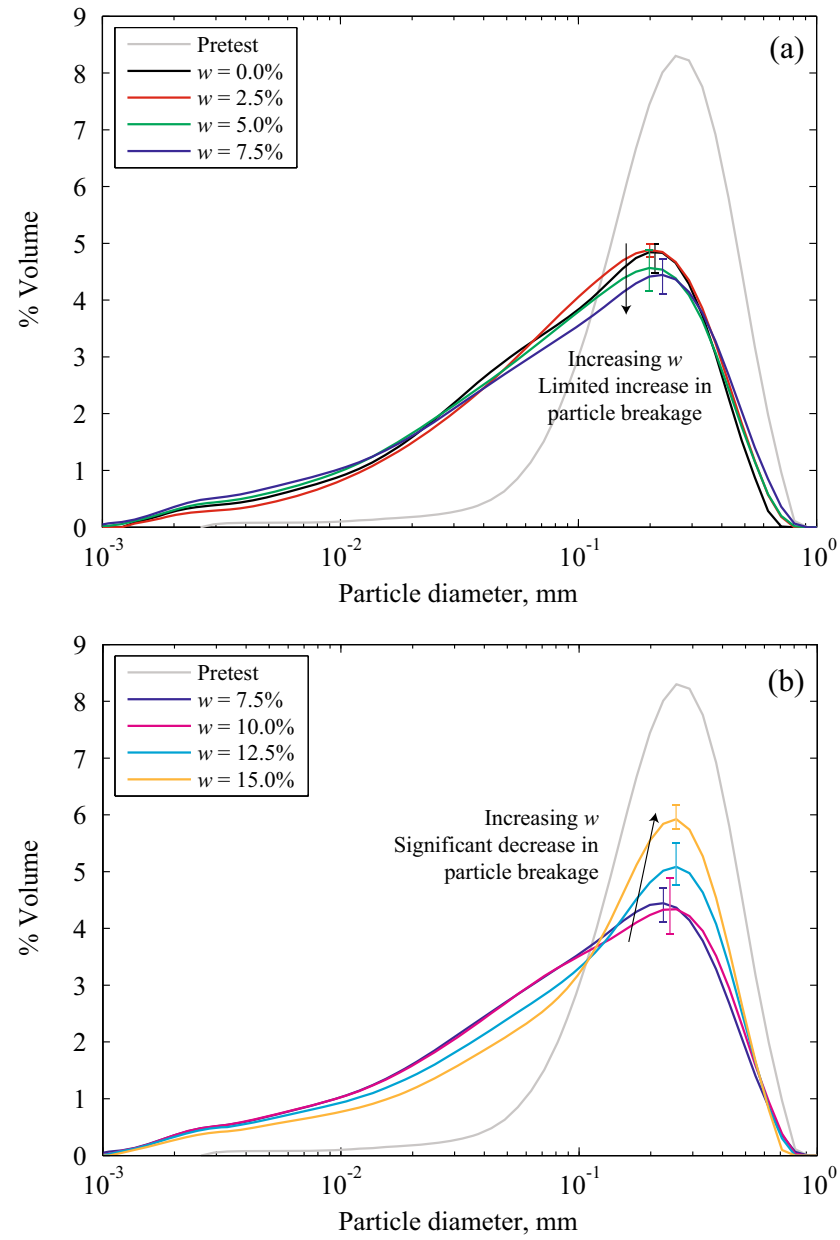

Fig. 4 Post-test particle size distributions for specimens with moisture contents of a $0.0 \%$ to $7.5 \%$, remaining partially-saturated throughout, and $\mathbf{b} 10.0 \%$ to $15.0 \%$, reaching full saturation during loading $(7.5 \%$ results repeated for reference). Each curve encloses a histogram with 50 log-spaced bins. Error bars indicate full range of results 


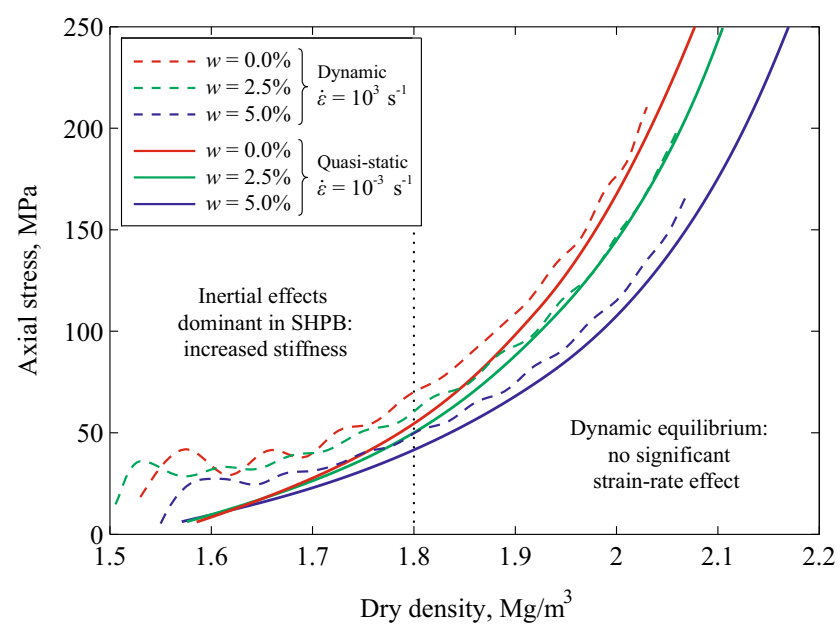

Fig. 5 Mean axial stress-dry density results from SHPB experiments at moisture contents of $0.0 \%$ to $5.0 \%$, compared with quasi-static experiments from Barr et al. [5]

Figure 5 shows the results of the $0.0 \%$ to $5.0 \%$ moisture content SHPB experiments compared with quasi-static experiments by Barr et al. [5] which used the same soil conditions. In this previous work inertial effects dominated the dynamic behaviour throughout SHPB loading, leading to an apparent strain-rate effect in measurements of axial stress. The more stable strain rate history in the current experiments reduced the inertial effects sufficiently to show that there is no significant strain-rate effect on stiffness between $10^{-3} \mathrm{~s}^{-1}$ and $10^{3} \mathrm{~s}^{-1}$.

In summary, this investigation used a SHPB to investigate the behaviour of loose quartz sand at moisture contents between $0.0 \%$ and $15.0 \%$, providing data for the modelling and development of effective soil-filled protective structures. The pre-saturation stiffness of the sand decreased as moisture content increased from $0.0 \%$ to $7.5 \%$, and then remained constant at higher moisture contents. The $10.0 \%$ to $15.0 \%$ specimens became fully saturated during the experiment, resulting in pore water loading and a significant reduction in particle breakage. Comparison with quasi-static experiments showed that there was no significant strain-rate effect on stiffness at these strain rates.
Acknowledgements This research was supported by the Engineering and Physical Sciences Research Council (EPSRC), award reference 1390522.

Open Access This article is distributed under the terms of the Creative Commons Attribution 4.0 International License (http:// creativecommons.org/licenses/by/4.0/), which permits unrestricted use, distribution, and reproduction in any medium, provided you give appropriate credit to the original author(s) and the source, provide a link to the Creative Commons license, and indicate if changes were made.

\section{References}

1. Felice C, Brown J, Gaffney E, Olsen J (1987) An investigation into the high strain-rate behavior of compacted sand using the split Hopkinson pressure bar technique. In: Proceedings of the 2nd symposium on the interaction of non-nuclear munitions with structures, pp 391-396

2. Veyera GE (1994) Uniaxial stress-strain behavior of unsaturated soils at high strain rates. Technical report, Wright Laboratory Flight Dynamics Directorate, AFMC

3. Luo H, Cooper WL, Lu H (2014) Effects of particle size and moisture on the compressive behavior of dense Eglin sand under confinement at high strain rates. Int J Impact Eng 65:40 55

4. Martin BE, Chen W, Song B, Akers SA (2009) Moisture effects on the high strain-rate behavior of sand. Mech Mater 41:786798

5. Barr AD et al (2016) Effects of strain rate and moisture content on the behaviour of sand under one-dimensional compression. Exp Mech 56(9):1625-1639

6. Clarke SD, Barr AD, Warren J, Williams A (2018) Local variations in gabion structures. Int J Protect Struct, in press

7. Dirlewanger $\mathrm{H}$ et al (2017) Enhancement of Hesco Bastion wall models to better predict magnitudes of response under far field loading conditions. In: 17th international symposium on the interaction of the effects of munitions with structures

8. Chen W, Ravichandran G (1997) Dynamic compressive failure of a glass ceramic under lateral confinement. J Mech Phys Solids 45(8):1303-1328

9. Tyas A, Pope DJ (2005) Full correction of first-mode PochammerChree dispersion effects in experimental pressure bar signals. Meas Sci Technol 16:642-652

10. Barr AD (2016) Strain rate effects in quartz sand. PhD thesis, The University of Sheffield

11. Shin YS, Lee M, Lam KY, Yeo KS (1998) Modeling mitigation effects of watershield on shock waves. Shock Vib 5(4):225234 